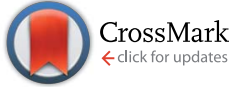

Cite this: J. Mater. Chem. A, 2015, 3 , 22739

Received 29th July 2015

Accepted 25th September 2015

DOI: $10.1039 / c 5 t a 05879 a$

www.rsc.org/MaterialsA

\section{A three layer design with mesoporous silica encapsulated by a carbon core and shell for high energy lithium ion battery anodes $\uparrow$}

\author{
Xi Cao, ${ }^{\text {ab }}$ Xiuyun Chuan, ${ }^{\text {aa }}$ Robert C. Massé, ${ }^{\mathrm{b}}$ Dubin Huang, ${ }^{\text {a }}$ Shuang Li ${ }^{\mathrm{b}}$ \\ and Guozhong Cao*b
}

A novel $\mathrm{C} / \mathrm{SiO}_{2}$ composite with a carbon-silica-carbon (C-mcms) three layer structure was synthesized and evaluated as an anode material for high-energy lithium ion batteries. The $\mathrm{C}$-mcms exhibits an excellent capacity of about $1055 \mathrm{~mA} \mathrm{~h} \mathrm{~g}^{-1}$ at a current density of $500 \mathrm{~mA} \mathrm{~g}^{-1}$ after 150 cycles without detectable decay, and high-rate capability, which is superior to other similar composites without the carbon core or the carbon shell. The results indicate the structural effect on the enhancement of the electrochemical properties: the $\mathrm{SiO}_{2}$ nanoparticles are completely confined between the carbon shell and the carbon core; the two carbon parts work together to keep the structural integrity and to keep the electrode highly conductive and active during cycling, which guarantees high capacity and cycle stability; the interior pores and voids among the $\mathrm{SiO}_{2}$ nanoparticles can provide free space to accommodate the volume change and buffer the volume effect of the electrode during lithiation/delithiation.

\section{Introduction}

As one of the most important candidates for the power source of portable electric devices, electric vehicles (EVs) and hybrid electric vehicles (HEVs), lithium ion batteries (LIBs) are attracting great interest due to their high energy density, long cycle life, and environmental friendliness. There is an increasing demand for rechargeable lithium-ion batteries with higher energy density and longer cycle life for applications to meet the rapid development in large-scale energy applications. ${ }^{1-5}$ Carbonaceous materials are commercially used as anode materials due to their advantages of long cycle life, safety and low cost. However, their low lithium-storage capacity $\left(\mathrm{LiC}_{6}\right.$ :

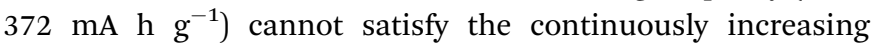
requirement nowadays. Various efforts have been made to developing new and better electrode materials with higher specific capacity and tailoring materials to new nanostructures in order to enhance their electrochemical performance. ${ }^{\mathbf{4 , 6 - 1 0}}$ Silicon based materials have attracted great interest due to the significantly higher theoretical specific capacity of about 4200 $\mathrm{mA} \mathrm{h} \mathrm{g}{ }^{-1}$ for the $\mathrm{Li}_{22} \mathrm{Si}_{5}$ alloy, low-cost and higher safety. However, the severe volume change (>300\%) occurring during

${ }^{a}$ School of Earth and Space Science, Peking University, Beijing 100871, PR China. E-mail: xychuan@pku.edu.cn; Fax: +86-10-62751159; Tel: +86-10-62767965

${ }^{b}$ Department of Materials Science and Engineering, University of Washington, Seattle, Washington 98195, USA. E-mail: gzcao@u.washington.edu; Fax: +1-206-543-3100; Tel: +1-206-616-9084

$\dagger$ Electronic supplementary information (ESI) available. See DOI: 10.1039/c5ta05879a the lithium insertion/extraction process leads to active particle cracking, electrode pulverization, and subsequent loss of electrical contact between the active material and current collector, resulting in severe capacity degradation. Several strategies have been proposed to deal with this problem, including the preparation of nanostructured $\mathrm{Si}^{\mathbf{1 1 - 1 5}}$ and composite materials. ${ }^{16-21} \mathrm{By}$ dispersing nano-Si particles in a carbon matrix, the electrochemical performance could be improved significantly. However, complex strategies are often required to prepare refined nanostructures, and the high cost of nano-silicon keeps the anode composite far from the industrial application level.

As a replacement, although having a specific capacity lower than that of Si (with the largest reversible capacity of $1961 \mathrm{~mA} \mathrm{~h}$ $\mathrm{g}^{-1}$; the theoretical capacity of $\mathrm{SiO}_{2}$ can be calculated according to different types of reactions that exist between silica and lithium ions proposed in a previous study, the detailed mechanism and calculation are listed in the $\left.\mathrm{ESI}^{22-25}\right), \mathrm{SiO}_{2}$ has also received a lot of interest due to its extremely low cost and less volume change during cycling. Even though lithium oxides and lithium silicates generated in the first discharge process act as buffer layers to accommodate the volume change of the amorphous Si particles to some extent, ${ }^{24-28}$ the volume effect, along with low initial coulombic efficiency and poor electrical conductivity still remains the main obstacles to enhancing the electrochemical performance. A lot of efforts have been made to address these problems, such as synthesizing silica materials with a carbon coating and different nanostructures. For example, Chang et al. ${ }^{23}$ reported that a $24 \mathrm{~h}$ high energy mechanically milled $\mathrm{SiO}_{2}$ would generate $5 \mathrm{~nm} \mathrm{Si}$ crystallites dispersed randomly within the amorphous silicon and exhibit 
a reversible capacity of $\sim 800 \mathrm{~mA} \mathrm{~h} \mathrm{~g}^{-1}$ at a current of 100 $\mathrm{mA} \mathrm{g}{ }^{-1}$ over 100 cycles. The amorphous $\mathrm{SiO}_{2} / \mathrm{C}$ composite prepared by mechanical milling and post heat-treatment exhibited a high reversible capacity of $600 \mathrm{~mA} \mathrm{~h} \mathrm{~g}{ }^{-1} \cdot{ }^{29}$ Hollow porous $\mathrm{SiO}_{2}$ nanocubes, hollow silica nanospheres and $\mathrm{SiO}_{2}$ nanotubes were also synthesized by a sacrificial template method and studied as anode materials. ${ }^{22,28,30}$ However, these methods may be time consuming, and cause environmental pollution and other issues. Thus, preparing $\mathrm{SiO}_{2}$ based composites with a controllable nanostructure and high capacity by a facile method is required for actual application.

In this work, a new type of $\mathrm{C} / \mathrm{SiO}_{2}$ composite with a carbonsilica-carbon three layer structure was prepared by a facile method, with mesoporous silica encapsulated by the carbon core and shell. When studied as an anode material for lithium ion batteries, this composite exhibited an excellent electrochemical performance with a high specific capacitance, good cycling performance and excellent rate capability. The significant impacts of the carbon core and carbon shell in enhancing the performance were systematically investigated.

\section{Experimental}

\subsection{Synthesis}

The fabrication of the $\mathrm{C} / \mathrm{SiO}_{2}$ composite with a carbon-silicacarbon three layer structure is illustrated in Fig. 1. Firstly, monodispersed mesoporous polymer nanospheres were prepared by a low-concentration hydrothermal method according to the literature. ${ }^{31}$ Secondly, $0.2 \mathrm{~g}$ of synthesized mesoporous polymer nanospheres was added into a solution containing $100 \mathrm{ml}$ of $\mathrm{H}_{2} \mathrm{O}, 60 \mathrm{ml}$ of ethanol and $300 \mathrm{mg}$ of cetyltrimethylammonium bromide (CTAB) and dispersed in an ultrasonication bath for $30 \mathrm{~min}$. Then, $1.65 \mathrm{ml}$ of ammonia (28 wt\%) was added into the mixture with mechanical stirring for another $30 \mathrm{~min}$. $0.5 \mathrm{ml}$ of tetraethyl orthosilicate (TEOS) was added into the above solution every 30 minutes and totally 3 times, and then the mixture was stirred for $12 \mathrm{~h}$. The assembled cationic surfactant micelle template (CTAB) serves as a structure-directing agent for polymerizing the silica component by electrostatic interactions. After the product was collected by centrifugation and washed with $\mathrm{H}_{2} \mathrm{O}$ and ethanol three times, respectively, the $\mathrm{CTAB}$ should be removed, and then, after calcination under an $\mathrm{Ar}$ flow at $500{ }^{\circ} \mathrm{C}$ for $3 \mathrm{~h}$ at a heating rate of $3{ }^{\circ} \mathrm{C} \mathrm{min}^{-1}$, a uniform core/shell mesoporous carbon-mesoporous silica (denoted as mcms) was obtained. Finally, $0.2 \mathrm{~g}$ of the obtained mcms was dispersed in a solution containing $10 \mathrm{ml}$ of $\mathrm{H}_{2} \mathrm{O}, 10 \mathrm{ml}$ of ethanol and $0.5 \mathrm{~g}$ of sucrose, and then transferred into a $30 \mathrm{ml}$ polytetrafluoroethylene (PTFE)-lined stainless steel autoclave and kept at $180{ }^{\circ} \mathrm{C}$ for $4 \mathrm{~h}$. The precipitate was collected and washed several times by centrifugation with deionized water after the autoclave was cooled down, and then dried at $80{ }^{\circ} \mathrm{C}$ overnight. The composites were annealed at $800{ }^{\circ} \mathrm{C}$ for $3 \mathrm{~h}$ under Ar flow to form the carbonsilica-carbon three layer structured composites (C-mcms).

In comparison, carbon core-mesoporous silica shell nanospheres without carbon coating on the surface (denoted as mcms) and the carbon coated hollow silica nanospheres (without the carbon core, denoted as C-ms) were also studied, as shown in Fig. 2.

\subsection{Structural analyses}

The crystal structure of the obtained sample was characterized by means of X-ray diffraction (XRD, D8 Bruker X-ray diffractometer with $\mathrm{Cu}-\mathrm{K} \alpha$ radiation $(\lambda=1.5418 \AA)$ within the range of $\left.10-80^{\circ}(2 \theta)\right)$. The morphology was examined using scanning electron microscopy (SEM, JEOL JSM-7000F). Transmission electron microscopy (TEM) measurements were conducted on a Tecnai F20 microscope (FEI) operating at $200 \mathrm{kV}$ accelerating voltage. The samples were suspended in ethanol and transferred onto a $\mathrm{Cu}$ grid for TEM measurements. The content of $\mathrm{SiO}_{2}$ was determined from the mass loss of the samples at $800{ }^{\circ} \mathrm{C}$ in air with a Q50 thermogravimetric (TGA) apparatus (TA, USA).

Nitrogen adsorption-desorption isotherms were measured using a Quantachrome NOVA 4200e system. The samples were degassed at $200{ }^{\circ} \mathrm{C}$ for $10 \mathrm{~h}$ under vacuum prior to measurements. The specific surface area and pore size distribution were determined by multipoint Brunauer-Emmett-Teller (BET), density functional theory (DFT) desorption analyses, respectively.

X-ray photoelectron spectroscopy (XPS) analysis was performed using a Surface Science Instruments S-probe spectrometer with a monochromatized $\mathrm{Al} \mathrm{K} \alpha \mathrm{X}$-ray and a low-energy electron flood gun for charge neutralization. The accelerating voltage and current were $40 \mathrm{kV}$ and $40 \mathrm{~mA}$, respectively. Three spots were tested on each sample to make the final results credible.

\subsection{Electrochemical measurements}

The electrochemical performance was tested in sandwich-type two-electrode coin cells. In order to prepare the working electrode, the as-prepared active materials were mixed and ground with Super P conductive carbon (TIMCAL graphite \& carbon),

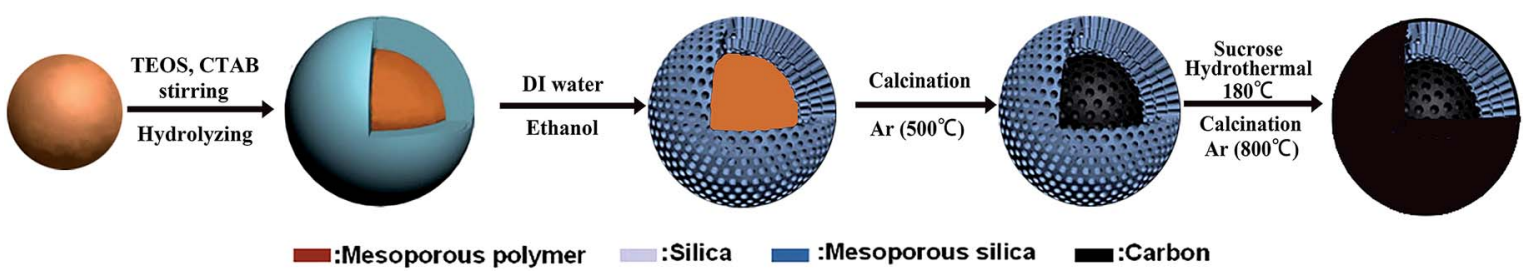

Fig. 1 Preparation of the carbon-silica-carbon three layer structured $\mathrm{C} / \mathrm{SiO}_{2}$ composite. 


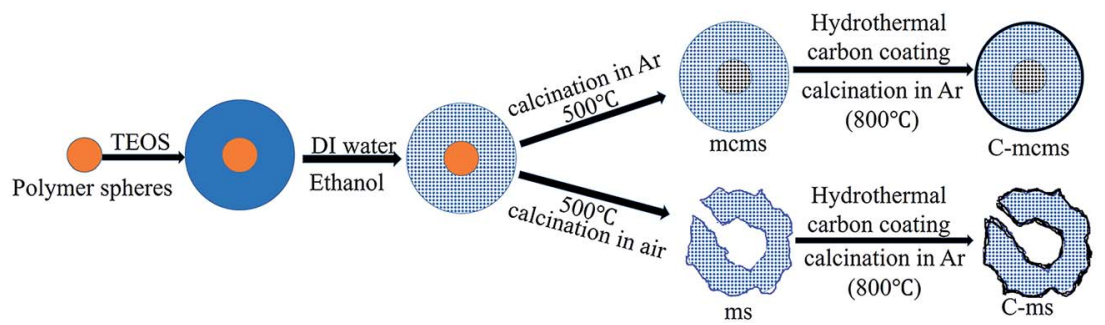

Fig. 2 Preparation procedure of the $\mathrm{mcms}, \mathrm{C}-\mathrm{mcms}$ and $\mathrm{C}$-ms composites.

and the poly(vinylidene difluoride) (PVDF, Sigma-Aldrich) binder dispersed in an $N$-methyl-2-pyrrolidone (NMP, Alfa Aesar) solution at a weight ratio of $80: 10: 10$, respectively. The as-prepared slurry was uniformly spread and pressed onto $\mathrm{Cu}$ foil and dried in a vacuum oven at $80{ }^{\circ} \mathrm{C}$ overnight as the working electrode. CR2016-type coin cells were assembled in an argon-filled glovebox (Innovative Technology, IL-2GB) with the working electrodes, pure lithium foil as the counter electrodes, and a polypropylene film as the separator. $1 \mathrm{M} \mathrm{LiPF}_{6}$ in a mixture of ethylene carbon (EC)/dimethyl carbonate (DMC) (volume ratio of $1: 1$, with $2 \%$ vinylene carbonate (VC) as the additive) was used as the electrolyte. All electrochemical measurements were performed with the assembled two-electrode battery at ambient temperature.

Galvanostatic discharge and charge measurements were performed with a LAND CT2001A tester (Wuhan, China) at different current densities in the voltage range of 0.01-3.5 V versus $\mathrm{Li}^{+} / \mathrm{Li}$. Cyclic voltammetry (CV) was conducted on an electrochemical analyzer (CH Instruments, model 605C) in the voltage range of $0.01-3.0 \mathrm{~V}\left(v s . \mathrm{Li} / \mathrm{Li}^{+}\right)$at a scan rate of $0.1 \mathrm{mV} \mathrm{s}^{-1}$. Alternating current (AC) impedance was recorded on the electrochemical analyzer ( $\mathrm{CH}$ Instruments, model 605C) with an amplitude of $5.0 \mathrm{mV}$ in the frequency range from $100 \mathrm{kHz}$ to $0.01 \mathrm{~Hz}$. The half-cells were tested at various current rates based on the weight of the active material alone.

\section{Results and discussion}

The XRD pattern of C-mcms shown in Fig. 3 demonstrates no distinct diffraction peaks, suggesting the amorphous feature of the $\mathrm{SiO}_{2}$. A broad and weak diffraction peak located in the $2 \theta$ range of $21-23^{\circ}$ should be associated with amorphous $\mathrm{SiO}_{2}$. Fig. 4 shows the high-resolution XPS spectra of $\mathrm{C}_{1 \mathrm{~s}}$ and $\mathrm{Si}_{2 \mathrm{p}}$ for

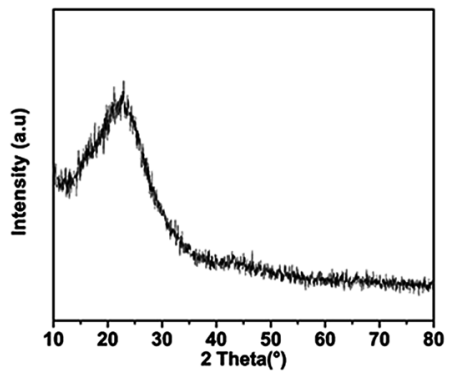

Fig. 3 XRD pattern of the C-moms composite.
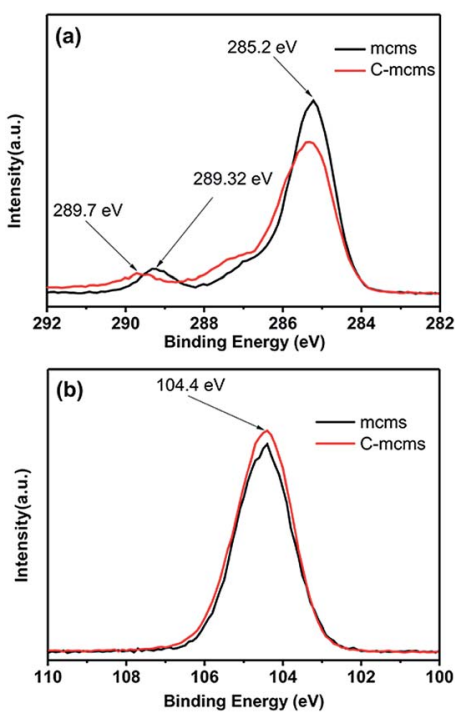

Fig. 4 High-resolution XPS spectra of $C_{1 s}$ (a) (inset: enlarged part of the $\mathrm{C}_{1 \mathrm{~s}}$ spectra) and $\mathrm{Si}_{2 p}$ (b) for $\mathrm{C}$-mcms and mcms composites.

samples mcms and C-mcms. The binding energy scales of the spectra were calibrated by assigning the most intense $\mathrm{C}_{1 \mathrm{~s}}$ peak a binding energy of $285.2 \mathrm{eV}$. As shown in Fig. 4a, compared with mcms, the intensity of the $\mathrm{O}-\mathrm{C}=\mathrm{O}(289.2 \mathrm{eV})$ peak decreases, and another peak at $289.7 \mathrm{eV}$ related to the asymmetric shape of non-graphic carbon ${ }^{32}$ appears when coated with carbon outside the $\mathrm{SiO}_{2}$. However, it is clearly indicated that there is only one peak at $104.4 \mathrm{eV}$ ascribed to $\mathrm{Si}^{4+}$ in the case of carbon coated mcms, as shown in Fig. 3b, suggesting the single amorphous phase of $\mathrm{SiO}_{2},{ }^{33,34}$ which demonstrates that $\mathrm{SiO}_{2}$ was not reduced to other forms of $\mathrm{Si}-\mathrm{O}$ composites.

TGA was used to determine the $\mathrm{SiO}_{2}$ content in the C-mcms, C-ms and moms composites, as shown in Fig. 5, and the $\mathrm{SiO}_{2}$ content reached $73.5 \%, 83.4 \%$ and $87 \%$, respectively.

Fig. 6 presents the general view of the morphologies of mcms, C-mcms, ms, and C-ms. Among them, moms exhibits a relatively uniform shape without agglomeration (Fig. 6a), and this structure remains after carbon coating on the surface (C-mcms, Fig. 6b). Comparatively, when mcms was converted to ms after calcination in air for $3 \mathrm{~h}$, some of the mesoporous silica (ms) spheres collapsed and broke into fragments due to the gas escape, and the uniform shape was also destroyed, as shown in Fig. 6c. Even after being coated with a layer of carbon, the same disordered morphology remained (C-ms, Fig. 6d), which may 


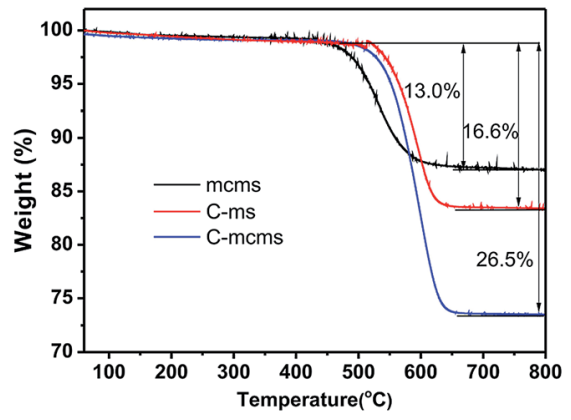

Fig. 5 The TGA curves of the $\mathrm{C}$-mcms, $\mathrm{C}$-ms and mcms composites from $25^{\circ} \mathrm{C}$ to $800{ }^{\circ} \mathrm{C}$ at a heating rate of $10^{\circ} \mathrm{C} \mathrm{min}-1$ in flow air.

result in a high surface area and surface energy. Lower magnification SEM images of these samples are shown in Fig. S1, $\dagger$ and the general views of the morphologies of these three samples are consistent with the above description.

Nitrogen adsorption measurements were used to determine the pore structure of the mcms, C-mcms, ms and C-ms composites. Fig. 7 shows their adsorption-desorption isotherms and the pore size distribution curves. All four samples exhibit type IV isotherms with a typical $\mathrm{H} 4$ type hysteresis loop, which is nearly parallel over an appreciable range of relative pressure, according to the IUPAC classification. The open $\mathrm{H} 4$ type hysteresis loop can be attributed to the mesoporous structures formed by slit-like pores and the aggregation of particles and internal voids in the spheres..$^{35}$ The core-shell structured mcms nanosphere sample shows a relatively narrow pore size distribution from $1.5-3 \mathrm{~nm}$, indicating the existence of mesopores which originated from the mesoporous silica shell and mesoporous carbon core. During the carbon coating process, some of the sucrose molecules (with a molecular diameter of about $0.9 \mathrm{~nm}$ ) were absorbed into these nanopores, and blocked them during carbonization, which resulted in the significant decrease of the detected specific surface area and pore volume. A similar phenomenon also happens for the C-ms sample. The surface areas of the coreshell mcms, C-mcms and C-ms are calculated to be 1226, 273 and $506 \mathrm{~m}^{2} \mathrm{~g}^{-1}$, respectively. The corresponding pore volumes are also calculated to be $0.30,0.17$ and $0.19 \mathrm{~cm}^{3} \mathrm{~g}^{-1}$, in which Cmcms demonstrates the lowest surface area and pore volume. The specific surface area and pore volume of the four samples are listed in Table 1.

As the density of $\mathrm{SiO}_{2}$ is $2.65 \mathrm{~g} \mathrm{~cm}^{-3}$, the average particle size of $\mathrm{SiO}_{2}(d)$ can be calculated based on the nitrogen-adsorption derived surface area.

$$
\begin{gathered}
S=\pi d^{2} \\
V=\pi d^{3} / 6 \\
S w=S / \rho V=6 / \rho d \\
d=6 / \rho S w=6 /(2.65 \times 106 \times 1188.0)=1.90 \mathrm{~nm}
\end{gathered}
$$

Transmission electron microscopy (TEM) was also carried out to provide further insight into the morphology and structure of the resulting C-mcms composites. As shown in Fig. 8, the carbon sphere core, porous silica layer, and carbon shell are organized compactly, which is in good accordance with the designed three layer structure. The size of each part of the C-mcms particle from inside to outside can also be measured to be about $100 \mathrm{~nm}$ diameter for the carbon core (the lighter area
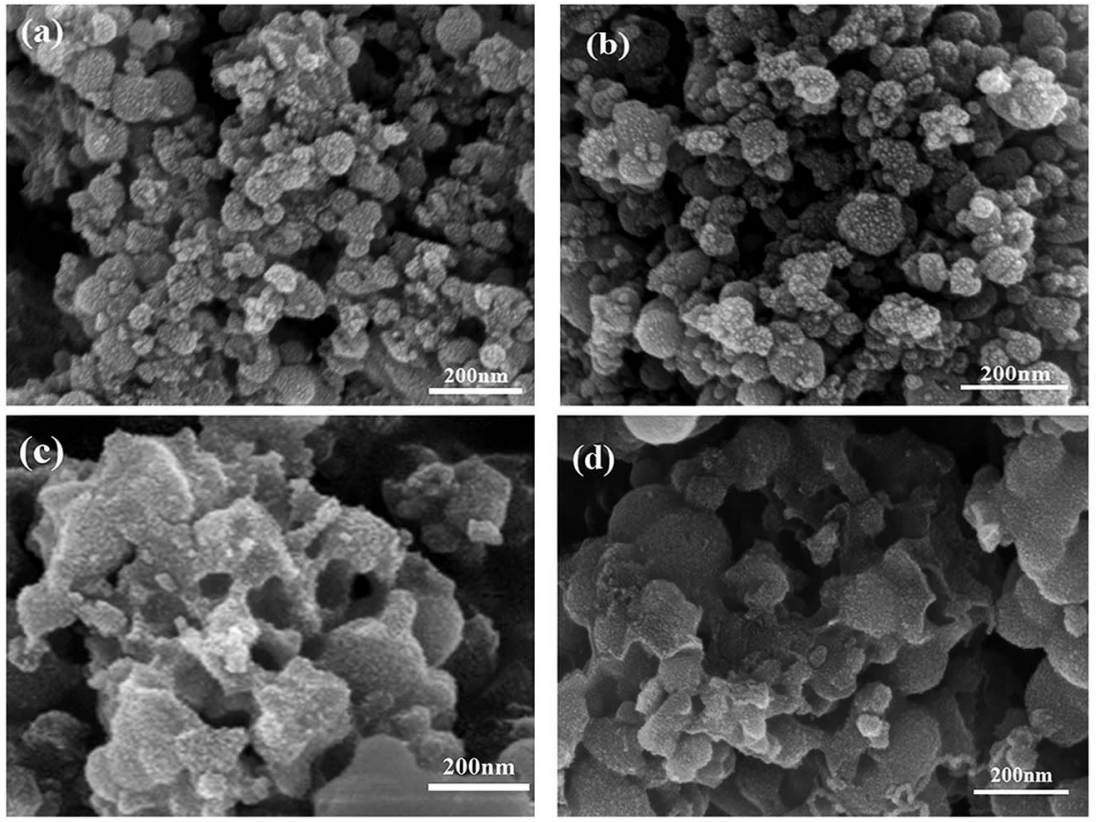

Fig. 6 SEM images of the carbon core-mesoporous silica without the carbon shell ( $\mathrm{mcms}$ ) (a), carbon core-mesoporous silica-carbon shell (Cmcms) (b), hollow mesoporous silica nanospheres without the carbon shell and carbon core (ms) (c), and hollow mesoporous silica nanospheres-carbon shell without the carbon core (C-ms) (d). 

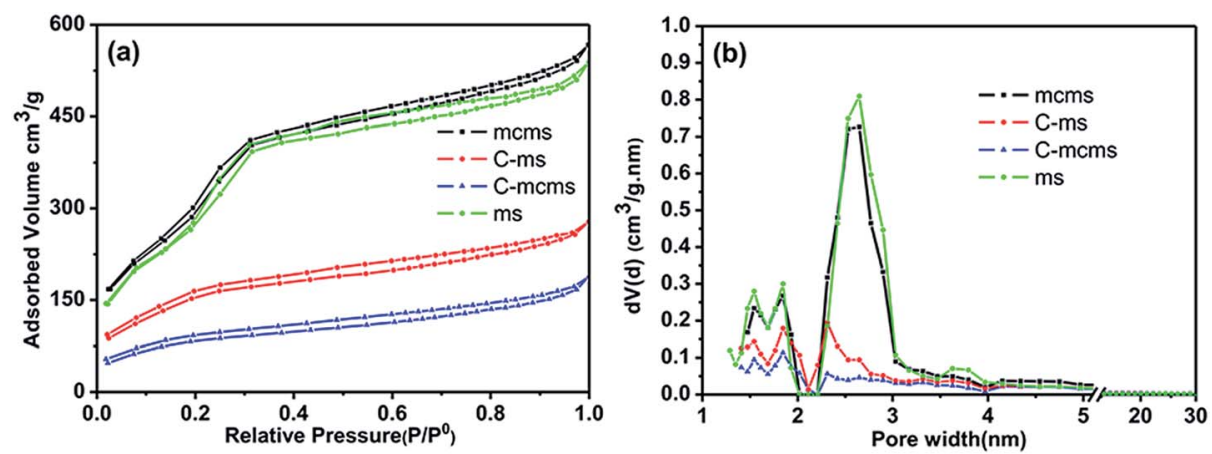

Fig. 7 Nitrogen adsorption-desorption isotherms (a) and the Density Functional Theory (DFT) pore size distribution (b) of mcms, C-mcms, ms and C-ms.

Table 1 The specific surface area and pore volume of the as-prepared C-mcms, C-ms, mcms, and ms

\begin{tabular}{lll}
\hline Sample & BET surface area $\left(\mathrm{m}^{2} \mathrm{~g}^{-1}\right)$ & Pore volume $\left(\mathrm{cm}^{3} \mathrm{~g}^{-1}\right)$ \\
\hline C-mcms & 273 & 0.17 \\
C-ms & 506 & 0.19 \\
mcms & 1226 & 0.30 \\
ms & 1188 & 0.27
\end{tabular}

inside), $50 \mathrm{~nm}$ thick for the mesoporous silica layer (the darker area) and about $6 \mathrm{~nm}$ thick for the carbon shell (the lighter layer outside, and the carbon layer of the C-mcms was also observed by HRTEM, and is shown in Fig. 8c). It can be clearly found that the whole C-mcms spheres demonstrate a uniform size of $200 \pm$ $10 \mathrm{~nm}$, and are also dispersed well without agglomeration.
STEM linescan was also carried out in order to further demonstrate the carbon-silica-carbon three layer structure of C-mcms. The Si, O, and $\mathrm{C}$ element distribution with the depth of the scanning point is shown in Fig. 8d. As we can see, there are no $\mathrm{Si}, \mathrm{O}$, and $\mathrm{C}$ elements detected at the start point. The $\mathrm{C}$ element shows a high content at the two edges of the scan line and the middle part of the particle, which refers to the carbon layer and carbon core, respectively. Correspondingly, Si and O elements show a higher content between the carbon layer and the carbon core, which refers to the $\mathrm{SiO}_{2}$ layer. Moreover, the $\mathrm{Si}$ and $\mathrm{O}$ element content decreases in the middle of the particle, with the increase of carbon content, which also confirms the three layer structure of C-mcms. TEM images of mcms and C$\mathrm{ms}$ are also shown in Fig. $\mathrm{S} 2 ; \uparrow$ the moms spheres demonstrate a uniform morphology and are also dispersed well without agglomeration (Fig. S2a †), which is similar to the C-mcms.
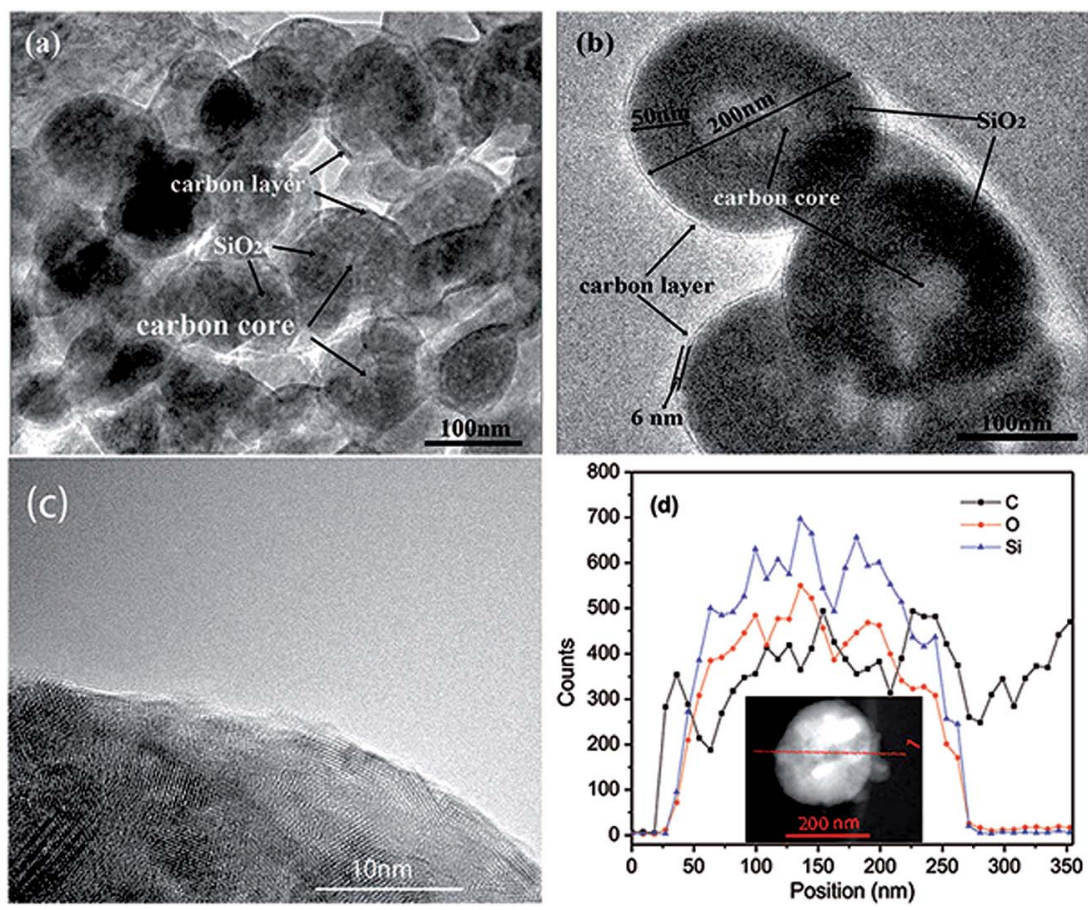

Fig. 8 TEM images of the C-mcms (a) and (b), HRTEM of the carbon layer (c), and STEM linescan of the three layer structure of C-mcms (d). 
However, the uniform structure collapsed and broke into fragments after calcination, and some parts of the C-ms particles also cannot be covered by the carbon layer, as shown in Fig. S2b, $\dagger$ which is consistent with the description of SEM images.

The electrochemical performance of the synthesized $\mathrm{C} / \mathrm{SiO}_{2}$ composites as anode materials for lithium ion batteries was evaluated using CR2016 coin-type half-cells in the voltage range of $0-3.5 \mathrm{~V}$.

The cyclic voltammetry (CV) curves of C-mcms at a scanning rate of $0.1 \mathrm{mV} \mathrm{s}^{-1}$ are displayed in Fig. 9. Several obvious reduction peaks located at $1.45 \mathrm{~V}, 1.26 \mathrm{~V}$ and $0.70 \mathrm{~V}$ can only be found in the first cycle. The small peaks at $1.45 \mathrm{~V}$ and $1.26 \mathrm{~V}$ may be attributed to side reactions between the electrolyte and electrode and the beginning formation of the solid electrolyte interface (SEI) layer on the electrode surface. The peak at $1.26 \mathrm{~V}$ may result from the reductive decomposition of vinylene carbonate (VC) as an additive in the electrolyte. ${ }^{36}$ According to the literature, the reduction peaks at around 1.0-1.5 V may be associated with the side reaction of $\mathrm{Li}^{+}$with the residual oxygencontaining groups in the carbon shell or carbon core, which also consumes lithium ions and results in irreversible capacities. $^{22}$ But the specific reaction between the electrolyte and electrode still remains unclear; more work may need to be done to explain it. While the long cathodic plateau at $0.70 \mathrm{~V}$ is associated with the electrolyte decomposition and the formation of SEI layer on the surface of the electrode..$^{22,30,36}$ As reported, the formation of the solid electrolyte interface (SEI) layer on the electrode surface may contribute a lot of the irreversible capacitance, and result in a low initial coulombic efficiency. And from the second cycle, all these peaks would disappear. The long cathodic peak below $0.5 \mathrm{~V}$ in the first cycle should be assigned to the electrochemical reduction of $\mathrm{SiO}_{2}$ to $\mathrm{Si}$, the irreversible formation of $\mathrm{Li}_{2} \mathrm{O}$ or $\mathrm{Li}_{4} \mathrm{SiO}_{4}$ (eqn (1a) and (1b)), and the insertion of lithium into active materials, including Si and carbon. ${ }^{29,36,37}$ These reactions can be summarized as follows:

$$
\begin{gathered}
2 \mathrm{SiO}_{2}+4 \mathrm{Li}^{+}+4 \mathrm{e}^{-} \rightarrow \mathrm{Li}_{4} \mathrm{SiO}_{4}+\mathrm{Si} \\
\mathrm{SiO}_{2}+4 \mathrm{Li}^{+}+4 \mathrm{e}^{-} \rightarrow 2 \mathrm{Li}_{2} \mathrm{O}+\mathrm{Si} \\
\mathrm{Si}+x \mathrm{Li}^{+}+x \mathrm{e}^{-} \leftrightarrow \mathrm{Li}_{x} \mathrm{Si}
\end{gathered}
$$

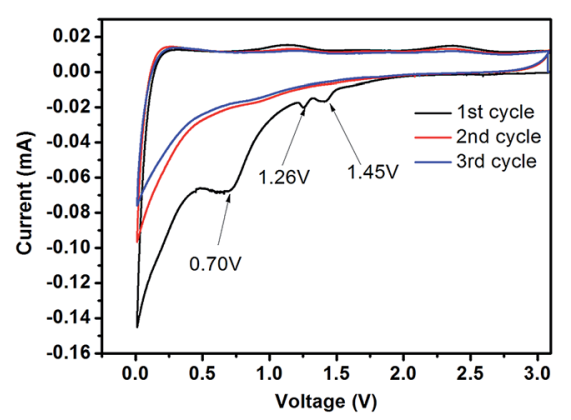

Fig. 9 Cyclic voltammetry profiles of the $\mathrm{C}$-mcms electrode measured in the voltage range of $0.01-3.0 \mathrm{~V}\left(\mathrm{vs}\right.$. $\left.\mathrm{Li}^{\prime} \mathrm{Li}^{+}\right)$with a scan rate of $0.1 \mathrm{mV} \mathrm{s}^{-1}$.

$$
\mathrm{C}+y \mathrm{Li}^{+}+y \mathrm{e}^{-} \leftrightarrow \mathrm{Li}_{y} \mathrm{C}
$$

Even though the inert $\mathrm{Li}_{2} \mathrm{O}$ and $\mathrm{Li}_{4} \mathrm{SiO}_{4}$ generated by reactions (1a) and (1b) contribute the major irreversible capacity, together with carbon, they can also act as a matrix to support and disperse the nano-Si particles preventing their aggregation and buffer the volume change during cycling, which is beneficial for maintaining a stable cycling performance, as evidenced by the following stable $\mathrm{CV}$ profiles after the first cycle. Both $\mathrm{Si}$ and carbon will alloy/dealloy with lithium reversibly and provide the reversible capacity (Reactions (2) and (3)) in the following cycles.

Fig. 10 displays the charge-discharge profiles of the three $\mathrm{C} / \mathrm{SiO}_{2}$ composites for the initial three cycles in the voltage range of 0.01 to $3.5 \mathrm{~V}$ at a current density of $100 \mathrm{~mA} \mathrm{~g}^{-1}$. Specifically, as shown in Fig. 10a, there are two small plateaus located at $1.45 \mathrm{~V}$ and $1.26 \mathrm{~V}$ and one relatively longer plateau located at $0.7 \mathrm{~V}$; these can only be found on the first discharge curve, which is consistent with the peaks in the CV profile. The initial discharge capacity of the C-mcms is found to be $2547 \mathrm{~mA}$ $\mathrm{h} \mathrm{g}^{-1}$, while the charge capacities reaches $1438 \mathrm{~mA} \mathrm{~h} \mathrm{~g}^{-1}$, with an initial coulombic efficiency of $56.5 \%$. By reducing the diameter of the $\mathrm{SiO}_{2}$ particles into nanosize, it may make the reaction more readily to complete in the first several cycles compared with the large particle size. The coulombic efficiency increases to $96 \%$ in the $2^{\text {nd }}$ cycle, and remains at approximately $100 \%$ in the following cycles. The high coulombic efficiency indicates a stable SEI film on the electrode surface. The coulombic efficiency climbing to above $90 \%$ in the second cycle is common in $\mathrm{SiO}_{2}$ (ref. 38 and 39) and other anode materials, for example, the $\mathrm{CE}$ of the $\mathrm{Si} / \mathrm{H}-\mathrm{rGO}$ composite film increases from $47 \%$ in the first coulombic efficiency to above $94 \%$ in the subsequent cycles. ${ }^{40}$ By reducing the diameter of the $\mathrm{SiO}_{2}$ particles into nanosize, it may make the reaction more easily to complete compared with the large particle size. However, the first charge capacity of C-ms and mcms only reaches 516 and $123 \mathrm{~mA} \mathrm{~h} \mathrm{~g}^{-1}$, and the corresponding coulombic efficiencies are calculated to be $33.8 \%$ and $18.6 \%$, respectively. Both the charge capacity and coulombic efficiency of C-mcms are much higher than those of $\mathrm{C}-\mathrm{ms}$ and mcms. According to previous reports, ${ }^{\mathbf{2 2 , 3 0 , 4 1 , 4 2}}$ the irreversible capacity loss in the first cycle is mainly ascribed to the formation of the solid electrolyte interphase and electrolyte degradation.

The rate performances of the three synthesized $\mathrm{C} / \mathrm{SiO}_{2}$ composites at various charge-discharge rates were also evaluated and compared. As shown in Fig. 11a, C-mcms was found to demonstrate a better rate performance compared to C-ms and mcms at each current density. For example, it demonstrates little difference in charge capacity between different current densities and presents a higher reversible capacity of approximately $1400 \mathrm{~mA} \mathrm{~h} \mathrm{~g}^{-1}$ at a current density of $100 \mathrm{~mA} \mathrm{~g}^{-1}$ and nearly $660 \mathrm{~mA} \mathrm{~h} \mathrm{~g}^{-1}$ even at a current density of $2000 \mathrm{~mA} \mathrm{~g}^{-1}$, demonstrating the excellent high rate performance for high power lithium-ion batteries. More significantly, the C-mcms electrode also displays a good capacity recovery when the current density switches back from $2000 \mathrm{~mA} \mathrm{~g}^{-1}$ to $100 \mathrm{~mA} \mathrm{~g}^{-1}$, 

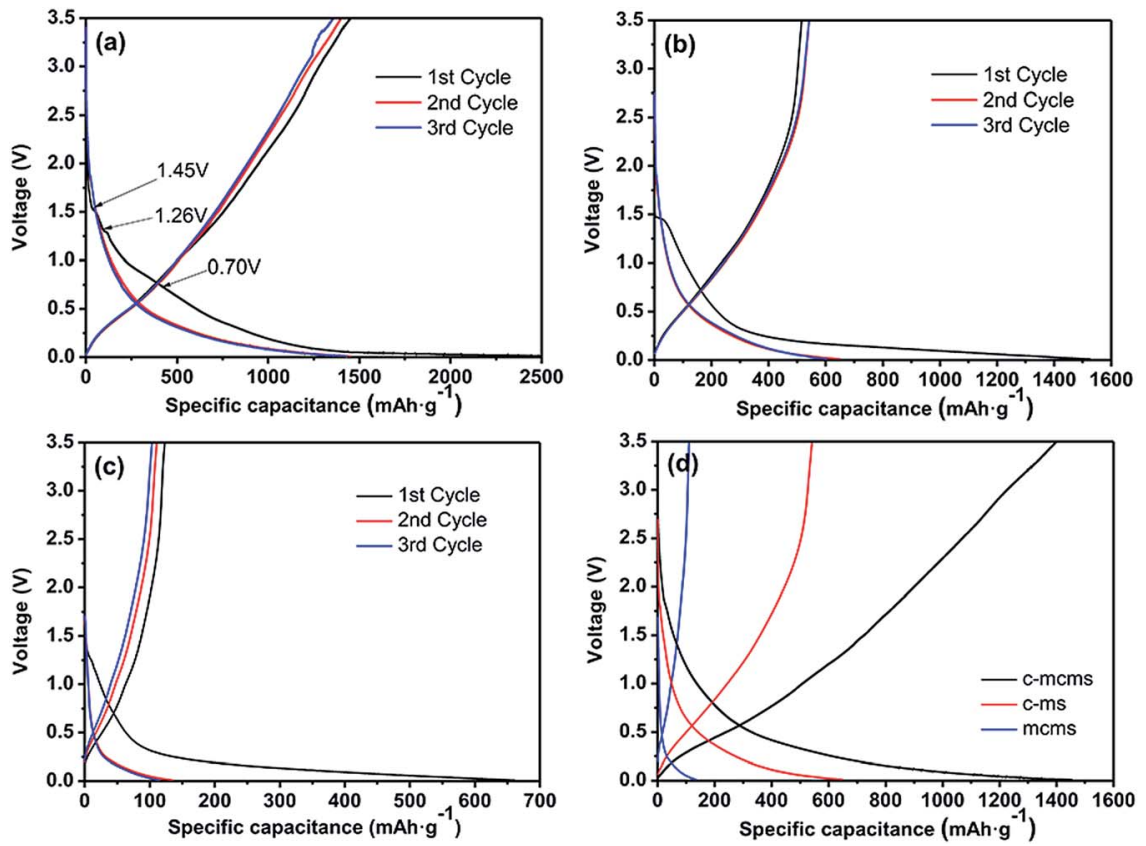

Fig. 10 First three discharge and charge curves of the C-mcms (a), C-ms (b) and mcms (c) electrode at a current density of $100 \mathrm{~mA} \mathrm{~g}{ }^{-1}$ and the comparison of $2^{\text {nd }}$ discharge and charge curves of the three samples (d).

suggesting good electrode structure stability. However, the other two electrodes, C-ms and mcms, deliver much lower capacities at different testing current densities, for example, C-ms delivers only $605 \mathrm{~mA} \mathrm{~h} \mathrm{~g}{ }^{-1}$ and moms delivers only a capacity of $110 \mathrm{~mA} \mathrm{~h} \mathrm{~g}^{-1}$ at a current density of $100 \mathrm{~mA} \mathrm{~g}^{-1}$. The rate performance of mesoporous silica was also studied and is shown in Fig. S3; $\dagger$ the capacity only reaches $115-120 \mathrm{~mA} \mathrm{~h} \mathrm{~g}^{-1}$ at a current density of $100 \mathrm{~mA} \mathrm{~g}^{-1}$ due to its low conductivity, which is similar to that of mcms. The results demonstrate that without the carbon coating on the surface of $\mathrm{SiO}_{2}$ particles, the conductivity and capacity would be extremely low.

The cycle performance of the three different samples was also evaluated for 150 cycles at a current density of $500 \mathrm{~mA} \mathrm{~g}^{-1}$, as shown in Fig. 11b; the C-mcms electrode delivers the highest reversible specific capacitance of more than $1055 \mathrm{~mA} \mathrm{~h} \mathrm{~g}^{-1}$, and can be maintained for 150 cycles without any tendency of degradation. According to the TGA result of C-mcms, considering the $26.5 \%$ percent of carbon (with the specific capacitance to be $130 \mathrm{~mA} \mathrm{~h} \mathrm{~g}^{-1}$, shown in Fig. 11a), it can be calculated that the specific charge capacity of the silica component is about $1388 \mathrm{~mA} \mathrm{~h} \mathrm{~g}{ }^{-1}$. However, the C-ms and mcms can only deliver a capacitance of $385 \mathrm{~mA} \mathrm{~h} \mathrm{~g}^{-1}$ and $55 \mathrm{~mA} \mathrm{~h} \mathrm{~g}^{-1}$ at a current density of $500 \mathrm{~mA} \mathrm{~g}^{-1}$, respectively. The three samples can all demonstrate a stable cycling performance, which suggests that a stable matrix was formed to support and disperse the nano-Si from aggregation and buffer the volume change during cycling.

Due to the absence of the carbon coating on the surface of mesoporous silica, the conductivity of mems could be very poor; it becomes relatively more difficult for the transportation of lithium ions throughout the silica particles, thus demonstrating a very low initial discharge capacity. Moreover, the high specific area of mcms indicates that the $\mathrm{SiO}_{2}$ is exposed to the electrolyte directly, resulting in more side reactions with the
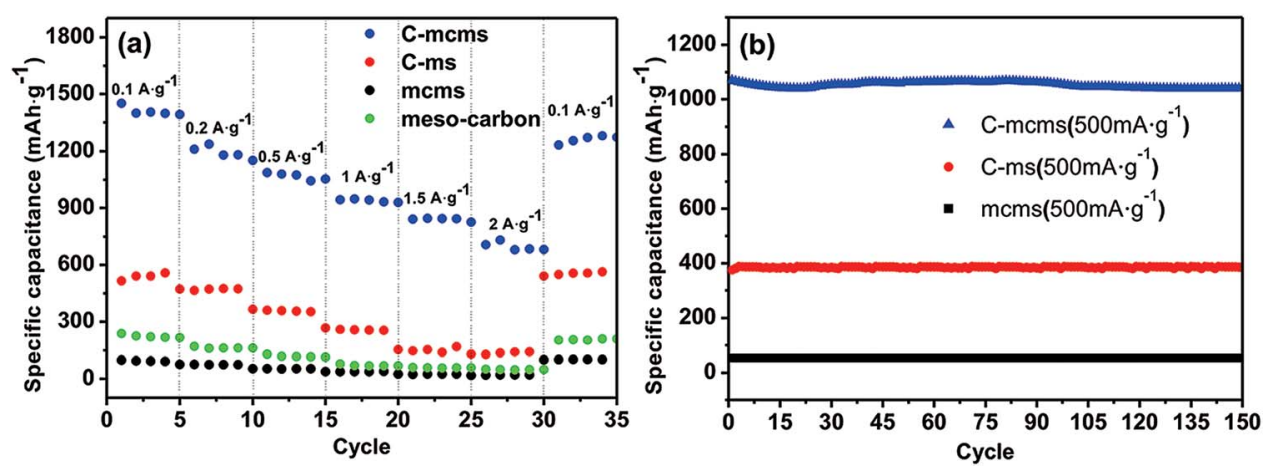

Fig. 11 (a) The comparison of the rate capability of the three samples and mesoporous carbon at different current densities and (b) the comparison of the cycling performance of the three samples at a current density of $500 \mathrm{~mA} \mathrm{~g}^{-1}$. 


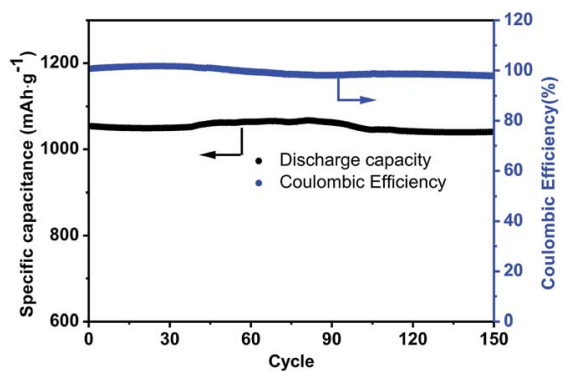

Fig. 12 Cycling performance of the $\mathrm{C}$-mcms electrode at a current density of $500 \mathrm{~mA} \mathrm{~g}^{-1}$

electrolyte to form excessive SEI layers on the surface of $\mathrm{SiO}_{2}$ particles and causing the loss of electrical connection with the active material, thus leading to poor coulombic efficiency and a big capacity decay, which is almost $80 \%$ of the first discharge capacity loss. For the C-ms with a carbon layer coated on the surface of hollow mesoporous silica spheres, rate and cycle performances are improved to a great extent compared with the moms. The conductive carbon framework can restrain the volume change of silica and guarantee rapid electron transfer throughout the composite. However, due to the structure collapse and deformation during calcination, some parts of the mesoporous $\mathrm{SiO}_{2}$ particles cannot be covered or filled by carbon, which results in the high surface area and pore volume (BET surface area: $506 \mathrm{~m}^{2} \mathrm{~g}^{-1}$ and pore volume: $0.19 \mathrm{~cm}^{3} \mathrm{~g}^{-1}$, respectively), thus lowering the integral conductivity. The uncoated or unfilled parts of $\mathrm{SiO}_{2}$ are still exposed to the electrolyte and it also results in the formation of an excessive SEI layer, thus lowering the reversible capacity.

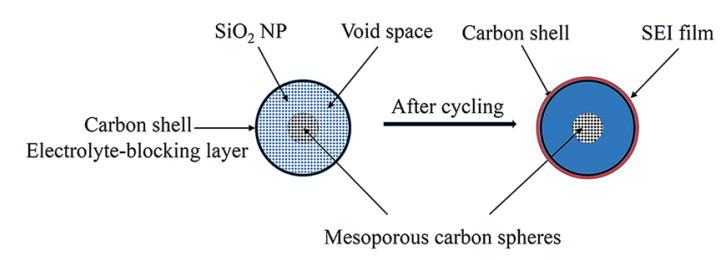

Fig. 13 Simplified two-dimensional cross-sectional view of one Cmcms sphere before and after electrochemical cycling.
In contrast, the C-mcms with a three layer structure exhibits the best cycle performances. Fig. 12 shows the cycling performance with a coulombic efficiency of the C-mcms electrode at a high current density of $500 \mathrm{~mA} \mathrm{~g}^{-1}$. As we can see, C-mcms demonstrates an excellent charge-discharge reversibility and superior cycling stability, with a high reversible capacity of more than $1055 \mathrm{~mA} \mathrm{~h} \mathrm{~g}^{-1}$ with the coulombic efficiency maintaining at nearly $100 \%$ during the cycles. The excellent electrochemical performance may mainly be ascribed to the refined structure design: firstly, the three layer structure ensures that the porous silica nanoparticles be completely confined between carbon core and the carbon shell, as shown in the TEM picture. The carbon core and carbon shell together act as a mechanical backbone to support the $\mathrm{SiO}_{2}$ nanoparticles to be electrochemically active and help to keep the structural integrity during cycling; secondly, the carbon coating on the surface of the mcms spheres and partly inside the silica nanopores, although just several nanometres thick, not only acts as an electrical highway to reduce the transport distance of electron/ lithium ions, but also lowers the surface area and results in less side reactions and less formation of the SEI. The carbon coated on the surface of $\mathrm{SiO}_{2}$ is also crucial for blocking the electrolyte outside the whole particle, minimizing the direct exposure of $\mathrm{SiO}_{2}$ particles to the electrolyte, and limiting majority of SEI formation to the outer surface of the carbon rather than the excessive formation on the $\mathrm{SiO}_{2}$ particles. Similar results have been reported before by encapsulating nano-silicon particles into a carbon chamber or hollow carbon spheres, and the electrochemical performance can be greatly enhanced. ${ }^{11,20,21}$ Thirdly, as the average particle size of $\mathrm{SiO}_{2}$ is calculated to be only $1.90 \mathrm{~nm}$, the ordered internal void space with an average diameter of $2.65 \mathrm{~nm}$ (from the PSD, Fig. 7b) among these nanoparticles can provide free space to accommodate the mechanical strain and allow the Si to expand without pulverization during the $\mathrm{Li}^{+}$intercalation and deintercalation process ${ }^{11,21,43}$ and help to form a relatively stable mixture, as illustrated in Fig. 13; the SEI layer formed outside of the carbon shell is helpful for maintaining a stable charge capacity and high coulombic efficiency. The assumption can be further confirmed by the SEM investigation of the C-mcms before and after cycling, as shown in Fig. 14; the C-mcms nanospheres
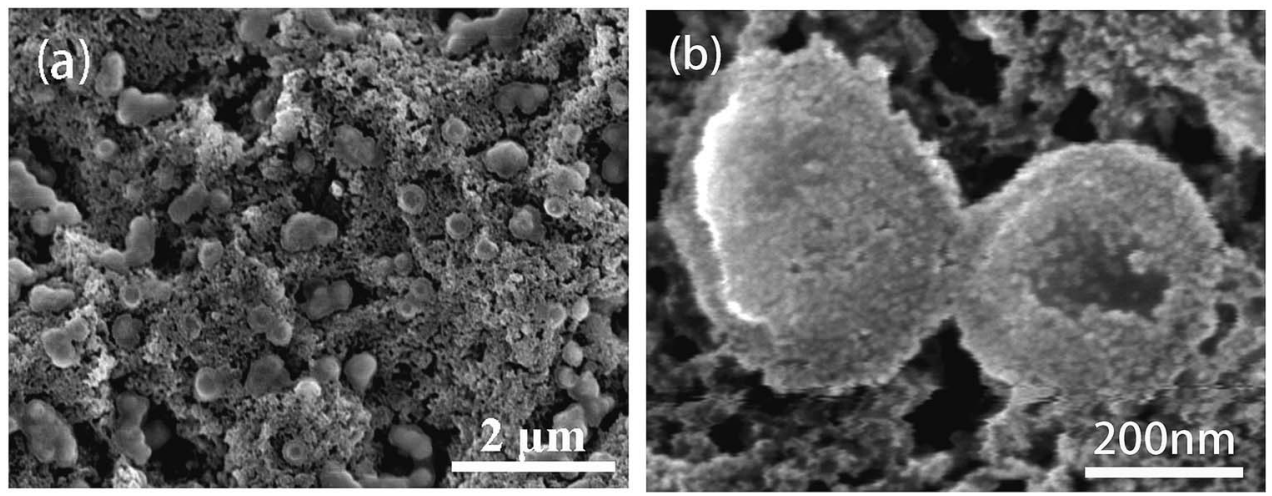

Fig. 14 SEM images of the C-mcms electrode after 150 cycles (a) and (b). 

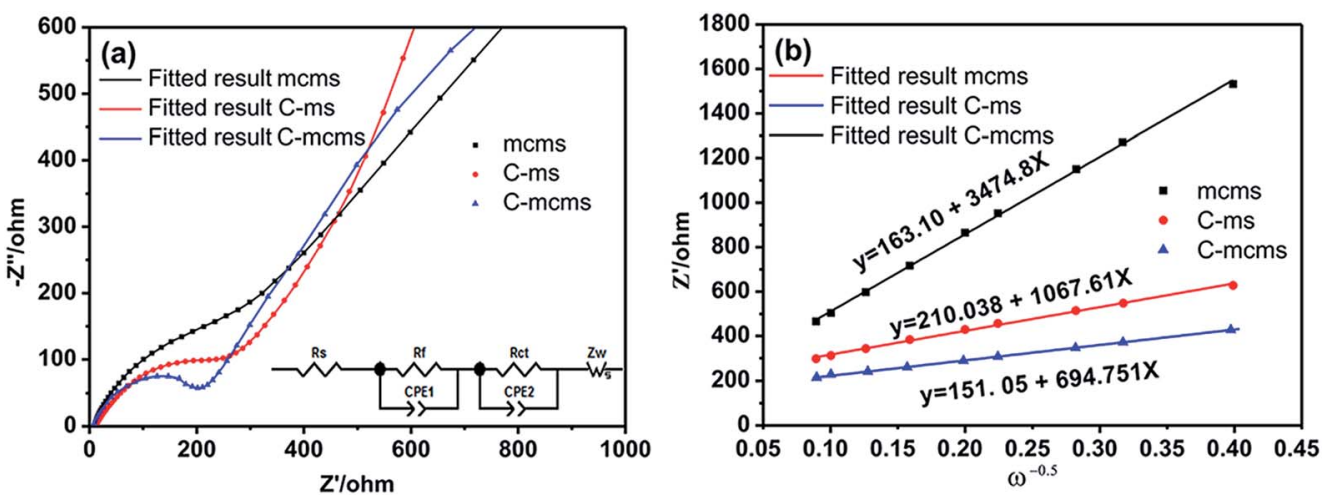

Fig. 15 (a) The EIS plots of the three samples at $25^{\circ} \mathrm{C}$ (inset: equivalent electrical circuit model) and (b) the $Z^{\prime}-\omega^{-0.5}$ plots in the low frequency range.

composite electrode did not crack and pulverize after 150 charge/discharge cycles. Fourthly, the inert $\mathrm{Li}_{2} \mathrm{O}$ and $\mathrm{Li}_{4} \mathrm{SiO}_{4}$ generated during the first lithiation process, together with the carbon layer, can also help to improve the cycle stability by buffering the big volume change caused by the lithiation of active $\mathrm{Si}$. Besides, by tailoring $\mathrm{SiO}_{2}$ particles into nanosize using the surfactant template, together with the nanopores among the $\mathrm{SiO}_{2}$ particles, the diffusion distance of lithium ions and electrons can be effectively reduced, thereby facilitating faster lithium ion transport in the silica matrix and ensuring an enhanced structural stability and cycle performance. ${ }^{22,30}$ At the same time, the local current density can also be decreased due to the high specific surface area of the silica nanoparticles. ${ }^{44}$

To further investigate the influence of different structures on the electrochemical performance of the three $\mathrm{C} / \mathrm{SiO}_{2}$ electrodes for lithium-ion energy storage, EIS measurements were performed over a frequency range of $100 \mathrm{kHz}$ to $0.01 \mathrm{~Hz}$. Fig. 15a shows the Nyquist plots of the three electrodes after 30 cycles to ensure the formation of stable SEI films. The resistance $R_{\mathrm{sf}}$ ascribed to the surface film and the charge transfer resistance $R_{\mathrm{ct}}$ due to the electrode reaction in the electrode-electrolyte interface are combined in a depressed semicircle in the high frequency region. ${ }^{45}$ The long slope line represents the Warburg impedance $\left(Z_{\mathrm{W}}\right)$ in the low frequency region, which indicates the diffusion of lithium ions into the solid matrix. The equivalent electrical circuit model (as shown in the inset of Fig. 13a) was used to fit the three Nyquist plots, in which the symbols $R_{\mathrm{s}}$, $R_{\mathrm{f}}, R_{\mathrm{ct}}$ and $Z_{\mathrm{W}}$ represent the solution resistance, contact resistance, charge-transfer resistance and Warburg impedance, respectively. The fitting results are listed in Table 2 . The $R_{\mathrm{f}}$ and $R_{\text {ct }}$ values of the C-mcms electrode are $103.6 \Omega$ and $68.2 \Omega$,

Table 2 Impedance parameters calculated from the equivalent circuit

\begin{tabular}{lccc}
\hline Sample & $R_{\mathrm{S}}(\Omega)$ & $R_{\mathrm{f}}(\Omega)$ & $R_{\text {ct }}(\Omega)$ \\
\hline mcms & 8.3 & 240.2 & 507.3 \\
C-ms & 11.9 & 120.5 & 264.3 \\
C-mcms & 7.02 & 103.6 & 68.2
\end{tabular}

respectively, which are the lowest compared with the other two electrodes. These results suggest that the special three layer structure with a carbon coating outside the mesoporous silica spheres lowers the contact and charge-transfer impedances, which is consistent with the above analysis. The lithium ion diffusion coefficient $\left(D_{\mathrm{Li}}\right)$ could be calculated from the low frequency plots based on the following equations:

$$
\begin{gathered}
Z^{\prime}=R_{\mathrm{e}}+R_{\mathrm{ct}}+\sigma \omega^{-0.5} \\
D_{\mathrm{Li}}=\frac{(R T)^{2}}{2\left(A n^{2} F^{2} C_{\mathrm{Li}} \sigma\right)^{2}}
\end{gathered}
$$

In eqn $4, \omega(2 \pi f)$ is the angular frequency in the low frequency region; both $R_{\mathrm{e}}+R_{\mathrm{ct}}$ and the Warburg coefficient $(\sigma)$ can be obtained from the fitting line while $Z^{\prime}$ has a linear relationship with $\omega^{-0.5}$. In eqn (5), $T$ is the absolute temperature, $R$ is the gas constant, $A$ is the surface area of the electrode, $n$ is the number of electrons transferred per mole of the active material involved in the electrode reaction, $F$ is Faraday's constant, and $C_{\mathrm{Li}}$ is the molar concentration of lithium ions. Based on the fitting linear equation in Fig. 15b, the lithium ion diffusion coefficients of C-mcms, C-ms and mcms are calculated to be approximately $1.19 \times 10^{-17} \mathrm{~cm}^{2} \mathrm{~s}^{-1}, 5.07 \times 10^{-18}$ and $4.79 \times 10^{-19} \mathrm{~cm}^{2} \mathrm{~s}^{-1}$, respectively. The lowest surface film and charge transfer resistance and higher lithium ion diffusion coefficient and electrical conductivity confirm that C-moms has more conductive pathways for lithium-ion transportation than the other two electrodes, resulting in enhancement in the electrochemical properties.

\section{Conclusions}

In summary, a new type of $\mathrm{C} / \mathrm{SiO}_{2}$ composite with a carbonmesoporous silica-carbon three layer structure was synthesized and evaluated as an anode material for high-energy lithium-ion batteries. The as-prepared composite with such a refined structure exhibits an excellent electrochemical capacity and cycle performance, and the effects of structure were systematically investigated by the comparison of similar composites 
without the carbon core or the carbon shell. It is revealed that the carbon shell may increase the integral electrical conductivity, decrease the SEI formation amount, and increase the initial coulombic efficiency, while the carbon core may support all $\mathrm{SiO}_{2}$ nanoparticles to be electrochemically active. Meanwhile, the mesopores in the $\mathrm{SiO}_{2}$ provided free space to accommodate the volume expansion and helped to keep a stable mixture during cycling. Therefore, with rational and optimal design of the $\mathrm{C} / \mathrm{SiO}_{2}$ composites, the C-mcms composite is able to demonstrate excellent reversible capacity, high rate capability and cyclic stability, and show great potential for application as the next generation anode materials for highenergy lithium-ion batteries.

\section{Acknowledgements}

This work was supported by the National Natural Science Foundation of China (grant No. 51274015) and the National Science Foundation (NSF-DMR 1505902). Xi Cao and Shuang Li would also like to thank the China Scholarship Council (CSC) for providing a scholarship for their Ph.D. study at the University of Washington.

\section{References}

1 J. B. Goodenough and K. S. Park, J. Am. Chem. Soc., 2013, 135, 1167-1176.

2 F. Cheng, J. Liang, Z. Tao and J. Chen, Adv. Mater., 2011, 23, 1695-1715.

3 H. Li, Z. Wang, L. Chen and X. Huang, Adv. Mater., 2009, 21, 4593-4607.

4 P. G. Bruce, B. Scrosati and J. M. Tarascon, Angew. Chem., Int. Ed., 2008, 47, 2930-2946.

5 B. Dunn, H. Kamath and J. M. Tarascon, Science, 2011, 334, 928-935.

6 Q. Zhang, E. Uchaker, S. L. Candelaria and G. Cao, Chem. Soc. Rev., 2013, 42, 3127-3171.

7 Y. Wang, H. Li, P. He, E. Hosono and H. Zhou, Nanoscale, 2010, 2, 1294-1305.

8 P. Poizot, S. Laruelle, S. Grugeon, L. Dupont and J. M. Tarascon, Nature, 2000, 407, 496-499.

9 W. W. Lee and J. M. Lee, J. Mater. Chem. A, 2014, 2, 15891626.

10 V. Augustyn, J. Come, M. A. Lowe, J. W. Kim, P.-L. Taberna, S. H. Tolbert, H. D. Abruna, P. Simon and B. Dunn, Nat. Mater., 2013, 12, 518-522.

11 N. Liu, Z. Lu, J. Zhao, M. T. McDowell, H.-W. Lee, W. Zhao and Y. Cui, Nat. Nanotechnol., 2014, 9, 187-192.

12 C. Yu, X. Li, T. Ma, J. Rong, R. Zhang, J. Shaffer, Y. An, Q. Liu, B. Wei and H. Jiang, Adv. Energy Mater., 2012, 2, 68-73.

$13 \mathrm{H}$. Wu and Y. Cui, Nano Today, 2012, 7, 414-429.

14 H. Wu, G. Chan, J. W. Choi, I. Ryu, Y. Yao, M. T. McDowell, S. W. Lee, A. Jackson, Y. Yang, L. Hu and Y. Cui, Nat. Nanotechnol., 2012, 7, 310-315.

15 H. Kim, M. Seo, M. H. Park and J. Cho, Angew. Chem., Int. Ed., 2010, 49, 2146-2149.
16 H. Lin, W. Weng, J. Ren, L. Qiu, Z. Zhang, P. Chen, X. Chen, J. Deng, Y. Wang and H. Peng, Adv. Mater., 2014, 26, 12171222.

17 K. Fu, Y. Lu, M. Dirican, C. Chen, M. Yanilmaz, Q. Shi, P. D. Bradford and X. Zhang, Nanoscale, 2014, 6, 7489-7495.

18 J. Chang, X. Huang, G. Zhou, S. Cui, P. B. Hallac, J. Jiang, P. T. Hurley and J. Chen, Adv. Mater., 2014, 26, 758-764.

19 R. Yi, F. Dai, M. L. Gordin, S. Chen and D. Wang, Adv. Energy Mater., 2013, 3, 295-300.

20 B. Wang, X. Li, X. Zhang, B. Luo, Y. Zhang and L. Zhi, Adv. Mater., 2013, 25, 3560-3565.

21 Y. Park, N. S. Choi, S. Park, S. H. Woo, S. Sim, B. Y. Jang, S. M. Oh, S. Park, J. Cho and K. T. Lee, Adv. Energy Mater., 2013, 3, 206-212.

22 N. Yan, F. Wang, H. Zhong, Y. Li, Y. Wang, L. Hu and Q. W. Chen, Sci. Rep., 2013, 3, 6.

23 W. S. Chang, C. M. Park, J. H. Kim, Y. U. Kim, G. Jeong and H. J. Sohn, Energy Environ. Sci., 2012, 5, 6895-6899.

24 Q. Sun, B. Zhang and Z. W. Fu, Appl. Surf. Sci., 2008, 254, 3774-3779.

25 B. K. Guo, J. Shu, Z. X. Wang, H. Yang, L. H. Shi, Y. N. Liu and L. Q. Chen, Electrochem. Commun., 2008, 10, 1876-1878.

26 C. Ban, B. B. Kappes, Q. Xu, C. Engtrakul, C. V. Ciobanu, A. C. Dillon and Y. Zhao, Appl. Phys. Lett., 2012, 100, 243905.

27 Y. Yao, J. J. Zhang, L. G. Xue, T. Huang and A. S. Yu, J. Power Sources, 2011, 196, 10240-10243.

28 M. Sasidharan, D. Liu, N. Gunawardhana, M. Yoshio and K. Nakashima, J. Mater. Chem., 2011, 21, 13881-13888.

29 P. P. Lv, H. L. Zhao, J. Wang, X. Liu, T. H. Zhang and Q. Xia, J. Power Sources, 2013, 237, 291-294.

30 Z. Favors, W. Wang, H. H. Bay, A. George, M. Ozkan and C. S. Ozkan, Sci. Rep., 2014, 4, 7.

31 Y. Fang, G. Zheng, J. Yang, H. Tang, Y. Zhang, B. Kong, Y. Lv, C. Xu, A. M. Asiri, J. Zi, F. Zhang and D. Zhao, Angew. Chem., 2014, 126, 5470-5474.

32 H. Estrade-Szwarckopf, Carbon, 2004, 42, 1713-1721.

33 Y. Liu, T. P. Chen, Y. Q. Fu, M. S. Tse, J. H. Hsieh, P. F. Ho and Y. C. Liu, J. Phys. D: Appl. Phys., 2003, 36, L97-L100.

34 S. Hofmann and J. H. Thomas III, J. Vac. Sci. Technol., B: Microelectron. Nanometer Struct., 1983, 1, 43-47.

35 K. S. W. Sing, D. H. Everett, R. A. W. Haul, L. Moscou, R. A. Pierotti, J. Rouquerol and T. Siemieniewska, Pure Appl. Chem., 1985, 57, 603-619.

36 Y. J. Du, M. Y. Hou, D. D. Zhou, Y. G. Wang, C. X. Wang and Y. Y. Xia, J. Energy Chem., 2014, 23, 315-323.

37 M. Q. Li, J. Li, K. Li, Y. Zhao, Y. G. Zhang, D. Gosselink and P. Chen, J. Power Sources, 2013, 240, 659-666.

38 P. Lv, H. Zhao, C. Gao, T. Zhang and X. Liu, Electrochim. Acta, 2015, 152, 345-351.

39 L. Zhang, J. W. Deng, L. F. Liu, W. P. Si, S. Oswald, L. X. Xi, M. Kundu, G. Z. Ma, T. Gemming, S. Baunack, F. Ding, C. L. Yan and O. G. Schmidt, Adv. Mater., 2014, 26, 45274532.

40 H. Tang, J. P. Tu, X. Y. Liu, Y. J. Zhang, S. Huang, W. Z. Li, X. L. Wang and C. D. Gu, J. Mater. Chem. A, 2014, 2, 58345840 . 
41 X. Q. Yang, H. Huang, Z. H. Li, M. L. Zhong, G. Q. Zhang and D. C. Wu, Carbon, 2014, 77, 275-280.

42 J. G. Tu, Y. Yuan, P. Zhan, H. D. Jiao, X. D. Wang, H. M. Zhu and S. Q. Jiao, J. Phys. Chem. C, 2014, 118, 7357-7362.

43 H. Zhong, H. Zhan and Y. H. Zhou, J. Power Sources, 2014, 262, 10-14.
44 P. Lv, H. Zhao, C. Gao, Z. Du, J. Wang and X. Liu, J. Power Sources, 2015, 274, 542-550.

45 M. E. Orazem and B. Tribollet, Electrochemical Impedance Spectroscopy, 2011. 